\title{
Specific laboratory investigations for assessments and management of drug problems
}

\author{
Kim Wolff, Sarah Welch \& John Strang
}

Much of the drug testing available today is able to determine the presence or absence of a variety of psychoactive substances in a range of body fluids and tissues. For the results of such tests to be confidently interpreted, additional information is required, including general assessment and historytaking. In a wide range of large psychiatric surveys, substance dependence emerges as one of the most common mental health-related disorders, and it is also the one that is least likely to be treated. The range of available tests can be best considered as acting to support and complement a broader assessment and diagnostic procedure.

The aim of this paper is to outline the variety of laboratory investigations available which could be considered as biological indicators of substance use and misuse. The strengths and weaknesses of different approaches and different body matrices are reviewed (urine, blood/plasma monitoring, saliva and hair testing), along with other possible materials not usually considered, with a focus on the clinical usefulness of these procedures.

A drug may be detected in any body fluid or tissue, but there are practical limitations to the extent to which samples can be and are used, and the mechanism of collection and supervision of samples are critical to the procedure.

\section{Chain of custody}

The procedure of ensuring that an identified sample was provided by a specified individual (chain of custody), and has subsequently been correctly labelled to ensure accuracy, must be properly documented. Chain of custody procedures usually require the collection of a urine sample witnessed by a designated member of staff (clinician, nurse, drug worker, therapist, etc.), and written confirmation of its validity from the individual voiding the sample, as detailed in Box 1 (Combs \& West, 1991).

\section{Legal and ethical issues}

The legal issues that have surfaced in the development of workplace drug testing programmes were reviewed by Long (1989). The legal cornerstone of

Box 1. Chain of custody procedures

Before, during and after urination, collection site personnel shall have the urine specimen in sight

Urine container shall be tightly capped and properly sealed and labelled with name and time date of collection

Approved chain of custody form shall be utilised to maintain accountability and accompany sample from initial collection to final disposition

Reliable transportation (courier system) to the laboratory is required

Kim Wolff is Academic Tutor \& Lecturer in the Addictions, Sarah Welch is a consultant psychiatrist and Honorary Senior Lecturer in the Addictions, and John Strang is Director of the National Addiction Centre (Institute of Psychiatry, Addiction Sciences Building, 4 Windsor Walk, Denmark Hill, London SE5 8AF). 
drug testing is a policy agreement which usually takes the form of a contract between employers and employees and may include the points listed in Box 2 (Osterloh \& Becker, 1990).

Those who oppose drug testing usually argue around issues concerned with civil rights. The courts have generally ruled that mandatory drug testing is a form of search and in some cases falls under constitutional protection. However, the potential value of testing to public health, safety and security in certain professions has led to wider legal support for testing programmes (MacDonald, 1990).

\section{Rationale}

The rationale for performing analytical tests varies depending upon the question(s) to be answered. Drug treatment services vary in the way that they practise drug screening. Services may use test results to gauge efficacy of therapy, or residential units requiring abstinence may use random checks of clients. Clinicians may also use drug tests to make the initial diagnosis of substance misuse, for screening as a requirement of a treatment programme when measuring compliance, or for screening as a useful adjunct to a full drug history to gauge drug exposure over time.

Major challenges for testing procedures include the vast array of drugs that may be consumed, the hugely different clinical and legal significance of test results (which may not always be obvious in the laboratory), and the need for specific approaches for these different substances. One of the limitations of drug testing is that it can give no indication of the presence or absence of physical dependence.

\section{Drug testing}

\section{Selecting the biological matrix and the challenge of interpretation}

The choice of body fluid is influenced by the pharmacokinetics of the drugs being tested for, and by the period of time that the clinician wishes to consider. Blood and, to a lesser degree, saliva are likely to give the most accurate measurement of drugs currently active in the system, whereas urine provides a somewhat broader time frame, but with less quantitative accuracy. Hair provides a substantially longer time frame.
Box 2. Drug testing policy agreement

The need for drug testing and why

Conditions of testing

Procedures for collecting specimens

Consequences of the test result

Availability of treatment

\section{Routine drug testing}

The standard procedure for routine analysis for drugs of misuse is an initial screening test using an automated commercial immunoassay kit such as an enzyme-mediated immunoassay (EMIT), followed by thin-layer, gas or liquid chromatography for confirmation of a specific drug in a sample (Braithwaite et al 1995; Simpson et al 1997). A confirmatory test is usually recommended (which should be qualitatively different from the first) following the initial screening test to specifically identify the compound detected because of the implications of a positive finding. Initial screening tests only identify the class of drug (i.e. opiate, benzodiazepine, etc.). The need to confirm the identity of the actual drug present will vary depending on whether this is in a legal, employment or clinical setting. The most sophisticated drug testing approach is gas chromatography coupled with mass spectrometry (GC-MS) which is currently regarded as the 'gold standard' by which to confirm the presence of a drug.

Although drug testing can be easily undertaken, there remains the problem of interpreting the results for clinical use. The picture is often complex; a drug may be present as metabolites and the parent drug may only be present in relatively low concentrations (buprenorphine, lysergic acid diethylamide (LSD) and $\Delta^{9}$-tetrahydrocannabinol) or not detected at all. Heroin (diamorphine), for instance, is seldom detected in blood as it is rapidly converted to an intermediary metabolite 6-monoacetylmorphine (MAM) and thus is not excreted into the urine.

Interpretation is also complicated by the relationship between compounds from the same drug class, which may share common metabolic end-products. For example, the benzodiazepine oxazepam, a prescription-only medication, is also a metabolic byproduct of chlordiazepoxide, diazepam, clorazepam and temazepam. A similar problem exists with the opiate drugs. The principle metabolite of heroin is morphine, but morphine is also a metabolite of codeine. Consequently, the detection of MAM is generally considered to be a more specific indication 
of heroin consumption. In contrast, dihydrocodeine (often confused with codeine) has its own distinct metabolic pattern. The clinical explanation of drug testing results is a challenging and often critical problem, particularly when establishing legitimate $v$. illegal use and all of its attendant complications.

\section{False positives and false negatives}

In qualitative screening tests, each sample is reported either positive or negative for a particular drug or drug group. There are four possible interpretations of the test result, as in Table 1.

The two true test results accurately reflect the clinical situation. However, a true positive on a screening test may not itself indicate the specific drug and cannot indicate the dose, time or route of drug administration. A true negative indicates that no drug was taken within the time desired for its use to be detected. Clinicians should be aware of the time taken for drugs to be eliminated from the body (see Table 2), since a negative test could result from not sampling soon enough after drug consumption. Clinically, a false negative may be defined as a negative finding in a sample from a patient known to have recently taken the drug of interest. False negatives can also occur when the threshold of sensitivity of the analytical procedure is set above the limit of detection of the drug.

Chemically, a false negative report may occur when the altered composition of a sample or endogenous components from the sample masks the presence of a drug of interest during analysis of a sample known to contain this substance. Aspirin ingestion and its presence in urine interferes with the analytical process in all EMIT assays, potentially yielding false negative results for drug screens (Linder \& Valdes, 1994). In the laboratory, a false positive test result arises when the positive test report is from the analysis of a biological sample which does not contain the drug in question. It is an incorrect identification of the presence of the determined compound. If the analytical method is subject to interference from artefacts or compounds

lable I Interpretation af tent renult

Person has Person has not taken drug taken drug

Test result positive True positive False positive

Test result negative False negative True negative
Table 2 Approximate duration of detectability of commonly used substances and some of their metabolites in urine (based on common laboratory cut-off values)

Substance Duration of detectability

\begin{tabular}{|c|c|}
\hline \multicolumn{2}{|l|}{ Stimulants } \\
\hline Amphetamine & $2-3$ days \\
\hline MDMA (ecstasy) & $30-48 \mathrm{~h}$ \\
\hline Methamphetamine & $48 \mathrm{~h}$ \\
\hline Cocaine $^{1}$ & $6-8 \mathrm{~h}$ \\
\hline $\begin{array}{l}\text { Cocaine metabolite/ } \\
\text { benzoylecgonine }\end{array}$ & 2 days \\
\hline \multicolumn{2}{|l|}{ Barbiturates } \\
\hline Short-acting (cyclobarbitone) & $24 \mathrm{~h}$ \\
\hline $\begin{array}{l}\text { Intermediate-acting } \\
\text { (pentobarbitone) }\end{array}$ & $48-72 \mathrm{~h}$ \\
\hline Long-acting (phenobarbitone) & $\geqslant 7$ days \\
\hline \multicolumn{2}{|l|}{ Benzodiazepines } \\
\hline Short-acting (triazolam) & $24 \mathrm{~h}$ \\
\hline $\begin{array}{l}\text { Intermediate-acting } \\
\text { (temazepam, chlordiazepoxide) }\end{array}$ & $40-80 \mathrm{~h}$ \\
\hline $\begin{array}{l}\text { Long-acting } \\
(\text { diazepam, nitrazepam })^{2}\end{array}$ & $\geqslant 7$ days \\
\hline \multicolumn{2}{|l|}{ Opiates } \\
\hline Methadone (maintenance dosing) & 7-9 days \\
\hline Codeine/morphine ${ }^{3}$ & $24 \mathrm{~h}$ \\
\hline Morphine glucuronides & $48 \mathrm{~h}$ \\
\hline Codeine glucuronides & 3 days \\
\hline $\begin{array}{l}\text { Propoxyphene/ } \\
\text { norpropoxyphene }\end{array}$ & $6-48 \mathrm{~h}$ \\
\hline Dihydrocodeine & $24 \mathrm{~h}$ \\
\hline Buprenorphine $e^{4}$ & $48-56 \mathrm{~h}$ \\
\hline Buprenorphine conjugates & 7 days \\
\hline \multicolumn{2}{|l|}{ Cannabinoids (marijuana) } \\
\hline Single use & 3 days \\
\hline Moderate use & 4 days \\
\hline Heavy use (daily) & 10 days \\
\hline Chronic heavy use ${ }^{5}$ & $\leqslant 36$ days \\
\hline \multicolumn{2}{|l|}{ Other } \\
\hline Methaqualone & $\geqslant 7$ days \\
\hline Phencyclidine (PCP) & 8 days \\
\hline Lisergic acid diethylamamide (LSD) ${ }^{6}$ & $24 \mathrm{~h}$ \\
\hline
\end{tabular}

1. Cocaine is rapidly converted to benzoylecgonine in alkaline urine at room temperature.

2. The presence of nordiazepam, an active metabolite of diazepam, in urine may prolong detection.

3. Morphine is rapidly oxidised at $4^{\circ} \mathrm{C}$. Urine collection vessels should be filled to the top to minimise this effect. 4. Usual doses results in very low levels of these compounds in urine. Detection may be possible after very recent use.

5. The lipophilic nature of the cannabinoids may prolong detection in urine with chronic dosing (Dackis et al, 1982).

6. LSD is extremely photolabile and samples thought to contain this drug should be stored protected from light. 
of similar structure to the drug of interest, then specificity is said to be low and this can lead to false positive results.

Clinical misinterpretations of immunoassay results (initial screening test) are a common source of false positive reporting. Immunoassays, as a measurement technique, are generally the most susceptible to interference from compounds similar to the drug in question, since they generally recognise all compounds belonging to a particular class of drug, including metabolites. An opiatepositive report using immunoassays, for instance, can be indicative of the presence of any number of opiate-type drugs and not necessarily illicit substances. Similarly, failure to acknowledge medication taken legitimately that is chemically similar to the drug of interest (e.g. pseudoephedrine in cough medicine with immunoassays for amphetamine) may lead to a false positive interpretation.

Clinically, a false interpretation of the result of a positive test can also arise because of passive drug exposure (cannabis or nicotine). Another cause of false interpretation of the result may be the presence (as already mentioned) of a compound which is actually a metabolite of other compounds. For example, the presence of morphine in urine is often assumed to be indicative of heroin use but it is important to recognise that urinary morphine may result from several sources (see Box 3). The 'poppy seed defence' has been shown to be a plausible explanation of a positive test result (as little as half a slice of poppy seed cake will give a positive immunoassay response). So much so that each case should be interpreted on its own merits and with caution (George, 1998).

Clearly, the more information that the clinician or drug worker can gather during sample collection, the better. Although self-reports of drug use have been criticised as an inaccurate source of information, carefully presented questions can produce a wealth of valuable information. Self-report interviews are all too often disregarded by workers in the field when they could be used as an important component of the screening test.

Box 3. Potential sources of urinary morphine

Consumption of codeine

Opiate drugs in foodstuffs, e.g. poppy seed strudel danish pastry (Selavka, 1991), poppy seed cake (George, 1998)

Analgesic prescription preparations, e.g. Gee's Linctus, kaolin and morphine mixture
Currently available laboratory investigations of drug use

Urine

Currently, urine is the preferred biological fluid for the screening of drugs of misuse (Wolff et al, 1999). Urinalysis is a well-known technology in which most of the analytical problems have been discovered and dealt with. The significant advantage of urine for drug testing is that it is generally available in sufficient quantity and the drugs or their metabolites tend to be present in relatively high concentrations (Moffat et al, 1986).

A variety of methodological techniques have been employed for screening urine samples, and many comparative studies have been carried out to determine the most efficient system (Wilson et al, 1994). Automation has enabled mass screening of urine samples by a variety of techniques. Enzymelinked immunosorbent assay (ELISA) is potentially the most cost-effective in terms of sample turnover and can be utilised for whole blood, serum, urine, saliva and hair (Simpson et al, 1997).

A recent innovation has been the introduction of self-contained drug testing kits for on-site testing. These are marketed for testing for a variety of drugs in urine and are designed to provide rapid access to test results without the need for laboratory facilities (Armbruster \& Krolak, 1992). Those that test for cannabis (see Jenkins et al, 1993, 1995) are among the most commonly used.

These tests offer advantages in terms of simplicity, ease of performance and rapid access to test results, but potential drawbacks include the subjective and qualitative nature of the kits and, in some instances, the lack of a positive control (Armbruster\& Krolak, 1992). There is a paucity of data on the validity of stick tests, which are produced by some manufacturers. The main problem with most self-contained urine tests is interpretation of the results (George \& Braithwaite, 1995). Detecting the colour change is a highly subjective process and difficult for the inexperienced eye. The other problem is the cost, which can be prohibitive. Test kits are best suited for testing small numbers, but are probably not suitable for widespread routine use.

It is often thought that detection times for drugs in urine will vary with drug dosage, but this is not usually the case (certainly not to any great extent) since chronic drug consumption only extends the detection time of the parent drug slightly. A threedays-per-week urinalysis schedule has been reported as the most efficient to pick up illicit cocaine or heroin use (Cone et al, 1992). However, the cost of 
conducting such frequent tests would be prohibitive for most services and is only recommended in exceptional circumstances.

The benefits of urine for screening for drugs of misuse are well known, but it has also been widely recognised that the collection of urine for drug screening has limitations. Care should be observed by those who collect urine for drug screening. For instance, morphine is rapidly oxidised at $4^{\circ} \mathrm{C}$ and urine collection vessels should be filled to the top, where possible, to minimise this effect.

Drug addicts may at times attempt to influence the results of a screening test to produce either a positive (prerequisite for pharmacotherapy) or negative (implied abstinent) test result. Various methods have been reported to achieve a false negative result. Household detergents added to urine prior to drug testing also cause false negative results for amphetamine, cocaine, morphine and cannabis, respectively. Ibuprofen in urine has been associated with false negative GC-MS confirmation results for cannabis (Brunk, 1988) while sodium chloride may lead to false negative results with basic drugs such as opiates (Kim \& Cerceo, 1976). Commercially available products for 'flushing' illicit substances from the body are used to achieve a negative result in a urine sample that is otherwise positive for one or more drugs (see Wu et al, 1994).

Other steps taken include drinking copious amounts of fluid to dilute the urine; substituting urine for weak black tea; providing a urine sample collected previously (when abstinent) or a sample from a drug-free friend; or diluting the sample by using water from a tap or the toilet (Widdop \& Caldwell, 1991). To help prevent dilution of the sample at the collection site, a blue dye can be added to the water in the toilet bowl. Similarly, manipulation to achieve a false positive result has been achieved by adding drugs to the urine sample after voiding (e.g. methadone detected in the absence of its main metabolite 2-ethylidene-1, 5-dimethyl-3, 3diphenylpyrrolidine (EDDP)). The best way to ensure sample validity is to witness the collection of the urine sample. This may raise several issues around infringements of a patient's personal privacy, more practical matters of staffing (i.e. female staff to observe female clients, etc.), and availability of facilities for such operations. There are, however, simple physical tests that may be taken to check the validity of a urine sample (see Table 3 ).

The disadvantages of using urine to test for the presence of drugs of misuse have to be weighed up against the advantages of having a fluid that requires little pre-analysis preparation and can be collected non-invasively in large volumes. Urinalysis remains the most reliable tool for identification of the presence of most illicit substances.

\begin{tabular}{|c|c|}
\hline $\begin{array}{l}\text { Suspected } \\
\text { manipulation }\end{array}$ & Physical test \\
\hline Dilute urine sample & $\begin{array}{l}\text { Measure specific gravity: } \\
\text { normal range } 1.016-1.025 \text {. } \\
\text { Sample dilute when } \leqslant 1.003\end{array}$ \\
\hline Dilute urine sample & $\begin{array}{l}\text { Measure creatinine level: } \\
\text { normal range } \\
\text { male } 1.5-2.0 \mathrm{~g} / 24 \mathrm{~h} \\
\text { female } 0.8-1.5 \mathrm{~g} / 24 \mathrm{~h} \text {. } \\
\text { Specimen dilute when } \\
\text { creatinine }<0.2 \mathrm{~g} / \mathrm{dl}\end{array}$ \\
\hline $\begin{array}{l}\text { Substituted sample } \\
\text { (tea/water/someone } \\
\text { else's urine) }\end{array}$ & $\begin{array}{l}\text { Check temperature is } \\
33-37^{\circ} \mathrm{C} \text { : measure within } \\
4 \mathrm{~min} \text {. of voiding }\end{array}$ \\
\hline Cold sample & $\begin{array}{l}\text { Check temperature is } \\
33-37^{\circ} \mathrm{C} \text { : measure within } \\
4 \mathrm{~min} \text {. of voiding }\end{array}$ \\
\hline $\begin{array}{l}\text { Adulterated sample } \\
\text { (bleach, detergents, etc.) }\end{array}$ & $\begin{array}{l}\text { Check pH: } \\
\text { normal range } 4.5-7.8\end{array}$ \\
\hline
\end{tabular}

\section{Blood}

Blood is the most useful biological matrix for the quantitative measurement of drugs and for interpretation by comparison with previously reported blood concentrations corresponding to therapeutic, toxic and fatal conditions (Moffatt et al, 1986). Also, because drugs leave the blood fairly rapidly, blood is most useful for identifying very recent drug use. Therapeutic levels of drugs generally exist in blood in low concentrations, typically in the $5 \mathrm{ng} / \mathrm{ml}$ to $5 \mu \mathrm{g} / \mathrm{ml}$ range. Some substances are found at lower concentrations in blood, notably LSD, flunitrazepam and buprenorphine. However, there is the advantage that when misused, drug concentrations in blood may be 2-3 times higher than normal levels observed with therapeutic dosing.

Since most drugs of misuse leave the blood fairly rapidly (within a few hours of drug use), blood concentrations (e.g. cocaine and ecstasy) will have fallen below detection levels applied in routine drug screening. The elimination of a drug from blood (half-life) is determined by the physiological and chemical characteristics of the particular drug and the route by which it is administered. It usually takes 5-7 half-lives for a drug to be totally eliminated from the body (see Table 4).

Until analytical assays are available to assess the biological effects of substance misuse, or until drug receptor concentrate can be estimated to indicate potential drug response, there is an increasing need 
for therapeutic drug monitoring (TDM) to predict clinical outcomes of therapeutic regimes (Flanagan, 1995). Blood collection, however, is probably the least favoured procedure for routine drug testing, and the mechanics of storage of blood samples collected for routine drug screening presents additional problems for the clinician (see Box 4).

Although clinical pharmacology is a wellestablished discipline, its influence on substance misuse treatment has been minimal. Dosage control has been the traditional tool for defining efficacy, safety and dose response in prescribing programmes for drug dependence. However, there is mounting evidence, particularly for methadone treatment, that a drug concentration strategy of treatment evaluation may represent a better approach to achieving optimal dosing (Wolff \& Hay, 1994).

\section{Monitoring blood levels in maintenance therapy}

Therapeutic drug monitoring is the science that combines measurement of blood drug concentrations with clinical pharmacokinetics. One of the major benefits of TDM is being able to monitor compliance with pharmacotherapy. The study of patient compliance is not new, and non-compliance is recognised to be common, occurring in all kinds of medical conditions of which drug dependence is no exception. Urinalysis drug screening is an important way of assessing illicit drug use by patients during methadone treatment but, unlike TDM, sheds no light on whether a patient is taking all their medication (at the correct time and in the correct amount), or is using extra medication (obtained illicitly), or is selling some of their prescription, perhaps to other users. There is growing evidence that plasma methadone measurements can provide answers to these questions (Wolff et al, 1992) (see Table 5).

Box 4. Issues to be overcome for blood collection

Requires trained personnel

Is an invasive procedure

Involves the attendant risk of needle-stick injury and possible transmission of either HIV or hepatitis B C virus

Is difficult to obtain in large volumes

Is difficult in intravenous drug users who may have thrombosed or sclerosed surface veins
Table 4 Approximate plasma elimination half-

lives for drugs of misuse

Drug Half-life (mean value)

Heroin $2 \mathrm{~min}$

Morphine

Morphine glucuronides

$3 \mathrm{~h}$

Dihydrocodeine

$7.5 \mathrm{~h}$

Codeine

Codeine glucuronides

$4 \mathrm{~h}$

$3 \mathrm{~h}$

Buprenorphine ${ }^{2}$

Buprenorphine glucoronides $^{2} \quad 24 \mathrm{~h}$

Methadone $^{3} \quad 36 \mathrm{~h}$

Amphetamine $\quad 12 \mathrm{~h}$

Cocaine $1 \mathrm{~h}$

Benzoylecgonine (cocaine metabolite) ${ }^{4} 7.5 \mathrm{~h}$

MDMA (ecstasy) $6 \mathrm{~h}$

Nitrazepam $28 \mathrm{~h}$

Flunitrazepam $25 \mathrm{~h}$

Temazepam $10 \mathrm{~h}$

Diazepam $48 \mathrm{~h}$

Nordiazepam ${ }^{5} \quad 40-100 \mathrm{~h}$

Cannabis $^{6} \quad 20 \mathrm{~h}$

Cannabinoid metabolites ${ }^{7} \quad$ 25-28h

1. Cone et al (1991).

2. Hanks (1987).

3. Wolff et al (1997).

4. Ambre (1985).

5. Nordiazepam is an active metabolite of diazepam Moffat et al, 1986).

6. Hunt \& Jones (1980)

7. Lemberger et al (1971) and Law et al (1984).

Studies of methadone treatment response have shown that patients who comply with the recommended course of treatment have longer-lasting post-treatment benefits (Allison \& Hubbard, 1985). Thus, it is discouraging for many practitioners that opiate addicts in treatment are frequently poorly compliant or non-compliant subjects. Dosage alterations based on interpretation of plasma measurements may help more patients to do well on methadone (Wolff et al, 1991a, 1997).

\section{Hair}

Human scalp hair testing is a fairly new biological tool for drug screening. The obvious advantage of hair testing is that this technique offers the potential for information over a much longer time-scale than can be obtained with blood, urine and saliva analysis. The analysis of misused substances in hair is problematic because these compounds are found in very low concentrations, usually within a range 
Iable : Indications for plasma methadone mearurements include:

\section{Decision to request plasma measurement}

\author{
Assessment of compliance with methadone \\ Monitor take home medication \\ Monitor prescription of ampoules \\ Suspect overscripting (higher dose than needed) \\ Suspect multiple scripting
}

Methadone induction change in dosage regime

Change in clinical state of patient

(i.e. pregnancy)

Potential drug interaction due to change in co-medications
Interpretation

Subtherapeutic concentrations

Not taking all of dose

Higher than expected concentrations

Consuming extra methadone

Subtherapeutic concentrations

Steady state may not have been achieved

\section{Subtherapeutic concentrations}

Increased blood volume in third trimester may reduce drug concentration with possibility of the onset of opiate withdrawal

\section{Subtherapeutic concentrations}

Enzyme inducing drugs (rifampicin, phenobarbitone, phenytoin, carbamazepine) will cause the onset of opiate withdrawal symptoms

\section{Toxic concentrations}

Enzyme inhibiting drugs (protease inhibitors - ritonavir, isoniazid, possibly cimetidine) may cause nausea or sedation

Subtherapeutic concentrations

Bingeing with alcohol may increase clearance and cause the onset of withdrawal symptoms of $10 \mathrm{pg} / \mathrm{mg}$ to $10 \mathrm{ng} / \mathrm{mg}$ hair. Nevertheless, most illicit substances have been detected in hair. Yet it is noticeable that there is little in the way of comprehensive drug screening methodology for this matrix.

Advocates of scalp hair analysis have emphasised the ease with which this matrix can be collected but there has been a tendency to gloss over the many technical and even practical difficulties involved (Cone, 1996). The mechanism by which a substance is deposited in hair remains unknown (Joseph et al, 1996), but it is likely that the amount and type of melanin is important in determining how much drug enters hair from the systemic circulation. This has led to claims that there may be a racial bias in hair analysis, but human studies to date have not confirmed this.

The physico-chemical properties of each particular substance play an important role in the incorporation of a drug into hair. Lipophilicity and basicity (alkalinity) are clearly important in the blood-to-hair route. Basic drugs (like opiates, amphetamine and cocaine) are incorporated easily into hair (Nakahara et al, 1995), acidic drugs (aspirin or methaqualone) much less so. Cannabis appears to be particularly difficult to detect in human scalp hair although analytical procedures have been reported (Kintz et al, 1995).

Perhaps a unique problem with scalp hair analysis is passive contamination of the external surface of the hair, which presents an obvious interpretational problem. Dupont \& Baumgartner (1995) demonstrated that exogenous false positives could be produced due to contamination of hair by drugs present in the environment. Decontamination of hair to exclude external interferants from analysis has become an integral step in most methodological procedures, but this process is very labour intensive and some hair types, such as thick black hair, seem more resistant to this process than others (Blank \& Kidwell, 1995).

While hair collection for drug screening is noninvasive there are certain precautions to be taken into account. Marsh (1997) reports that maintaining alignment of, securing and identifying the cut ends of hair are critical if segmentation (quantification of time with drug concentration) is required. The style of hair worn is also a source of variability. Hair growing from a closely cropped head can not 
possibly contain the same concentration, after the same dose of drug, as hair that has not been cut over a long period of time. Variations in the expected concentration can exceed $20 \%$ (Sachs, 1995), and it is not clear from the literature how one corrects for these discrepancies. Additionally, some patients wear wigs, and hair from other parts of the body is needed for these and for people with shaved heads.

In regular practice in the addiction field, the clinician may be better served by urinalysis (Strang et al, 1993). Hair analysis should be seen as an adjunct to other screening procedures, for obtaining information regarding patterns of drug use, for establishing a drug history over time, or as a research tool, for example, in investigation of passive exposure to tobacco.

\section{Likely future development of new laboratory investigations}

\section{Saliva}

Determination of drug concentrations in saliva is an exciting new territory, particularly since the collection of samples is non-invasive, and this matrix has the potential to provide both quantitative and qualitative information on the drug status of an individual under examination. Measurements of illicit drugs have been investigated in saliva for more than a decade, but early work was hampered by inconsistent collection procedures (Haeckel \& Hanecke, 1996). For the moment, the role of this biological fluid as a detection and monitoring tool remains uncertain.

Sample collection time is also an important variable. For drug addicts who self-administer by

Box 5. Additional potential uses of hair analysis

In post-mortem examination (Kintz et al, 1992)

Forensic cases (Moeller, 1996)

Medico-legal work in establishing a history of past exposures to therapeutic or abused substances (Huestis, 1996)

Hair samples from children may help in child protection cases by providing evidence of previous drug exposure (Lewis et al, 1997)

Neonatal hair analysis may (if present in sufficient quantity) be a useful biological marker for foetal exposure to drugs (Klein et al, 1994) oral, intranasal and smoking routes, 'shallow depots' of drug can result, which contaminate the oral cavity (Cone, 1993). This produces elevated salivary concentrations for several hours after ingestion and, although potentially useful in cases of overdose or accidental poisoning (Wang et al, 1994), should be borne in mind when interpreting results for routine drug screening. The presence of tetrahydrocannabinol in saliva, for example, appears to be due primarily to contamination of the oral cavity following smoking of marijuana cigarettes. Being highly protein-bound, cannabinoids do not readily pass from blood into saliva and cannabis itself inhibits salivary excretion (Karlsson \& Strom, 1988).

Saliva testing for drugs of misuse can, however, provide information on the drug status of an individual undergoing testing. Generally, once drugs have been eliminated from the buccal cavity, there is a high correlation between salivary and plasma drug concentrations for many compounds (Huestis \& Cone, 1998) such as cotinine (Curvall et al, 1990) and methadone (Wolff et al, 1991b). Saliva for drug analysis has the advantage of being relatively easy to obtain and collection can be carried out by nonclinical personnel (Gorodischer et al, 1994). Commercially available tools may make possible the collection of saliva in a more standardised manner than has been possible previously. This would enable more frequent use of saliva in substance misuse treatment services, which would be particularly advantageous in intravenous drug users in whom it is often extremely difficult to find venous access. It would also enable more objective measurement and corroboration of self-reported drug use in a variety of social survey situations.

Despite the fact that drugs are detectable in saliva for a slightly shorter period than in urine, it is possible that salivary detection procedures will expand significantly in the next few years owing to the particular advantage over blood of the noninvasive nature of collection.

\section{Breath}

The analysis of human breath has been extensively used for the measurement of ethanol as a gauge of alcohol consumption (Cowan et al, 1996). Generally, the variety of instruments used to monitor breath are portable, economical and easy to use for patient sampling (Grote \& Pawliszyn, 1997). Some drugs are naturally volatile compounds that mix freely with air, such as anaesthetic gases (e.g. nitrous oxide) and inhalants (e.g. correction fluid and solvents). Others require combustion (e.g. tobacco, marijuana and crack cocaine). Although tetrahydrocannabinol was identified in the breath of marijuana 
smokers by using radioimmunoassay and gas chromatography over a decade ago (Manolis et al, 1983), procedures for drug testing using breath remain underdeveloped. The increasing sophistication of such technology is likely to improve both the availability and accuracy of such tests for wider clinical application.

\section{Sweat}

In recent years, remarkable advances in the sensitivity of analytical procedures have enabled the analysis of drugs on unconventional samples such as sweat. Sweat is collected by means of a 'sweat patch' applied to various sites of the skin (biceps, torso or back). All of the commonly used illicit substances, including buprenorphine and ecstasy, have been detected in sweat using precision chromatographic techniques (Kintz et al, 1996a; Skopp et al, 1996). Recently, sweat patches have been employed in drug treatment services to monitor patients prescribed methadone and have been reported to compare favourably with urine drug tests (Taylor et al, 1998; Kintz et al, 1998). Intrasubject variability and the influence of site application have been reported to be significant (Kintz et al, 1996b), and it is possible to adulterate the sweat patch by injection into or under the patch to cause a false positive (Fay et al, 1996) or false negative (Fogersom et al, 1997) test response.

However, little information is available on the characteristics of drug excretion in sweat under controlled dosing conditions, and sweat analysis is open to misinterpretation because of the difficulty of excluding topical contamination. Given the reported between-dose and between-subject variability, sweat patch test results cannot as yet be used to determine either the dose or the time of use (Burns \& Baselt, 1995). Nevertheless, the sweat test does appear to offer an alternative non-invasive means of obtaining a cumulative estimate of drug exposure over a period of 7-21 days (Kintz, 1996). From a clinical viewpoint the sweat patch could serve as a useful monitoring device in surveillance of individuals on treatment and probation programmes. If applied to the skin with a tamper detection device, it could provide a more continuous monitor of drug exposure.

\section{Body fluids and childbirth}

The high increase in the number of pregnant substance users presenting at drug treatment agencies for treatment for their addiction, and at antenatal clinics, has led to a demand for improved technology to assess the extent of drug ingestion by the foetus and/or neonate.

\section{Breast milk}

All drugs pass, to some extent, from plasma into human breast milk. The milk-to-plasma concentration ratio is the most commonly used index of drug distribution into milk, and is used to calculate the likely infant drug dose from a given maternal plasma concentration (Atkinson \& Begg, 1990). The usefulness of human breast milk for assessing the extent of infant exposure to illicit substances has not been fully evaluated (Huestis \& Cone, 1998). In Britain, drug-dependent nursing mothers are usually advised against breast-feeding. In many cases, this may be unnecessary as the total dose to which the baby is likely to be exposed is thought to be negligible (Atkinson \& Begg, 1990). A particularly important case for consideration may be nursing mothers prescribed methadone. Breast-feeding has been advocated for methadone-maintained women (Batey et al, 1990), and may assist in reducing the severity of neonatal abstinence syndrome (Mack et al, 1991), although polydrug use is likely to complicate decisions made regarding suitability for breast-feeding.

Reports indicate accumulation of tetrahydrocannabinol (Astley \& Little, 1990), amphetamine (Steiner et al, 1984) and cocaine (Dickson et al, 1994) in breast milk. Steiner et al (1984) argued 15 years ago that analysis of breast milk for drugs of misuse should be performed in cases of possible infant intoxication, but such tests have yet to become routinely available. Further research in this area is required since the extent to which the ingestion of illicit and psychotropic drugs by nursing infants may affect growth and development is unknown.

\section{Meconium}

Many substances have been detected in meconium including cannabis (Moore et al, 1996), methadone (Skolk et al, 1997), nicotine (Eliopoulos et al, 1996) and cocaine (Martin et al, 1996). However, analysis of drugs in meconium is currently only conducted by researchers.

\section{Conclusion}

Analysis of urine is currently the biological tool of choice for qualitative detection of illicit drug use. Quantitative accuracy usually demands the collection of a blood sample, although saliva may be an alternative in the future. The advantage of hair 
sampling is its reflection of weeks rather than hours of recent use. Accurate interpretation of the screening tests within a clinical setting, alongside other relevant information, remains the key to the usefulness of any test.

\section{References}

Allison, M. \& Hubbard, R. I. (1985) Drug abuse treatment process: a review of the literature. International Journal of Addiction, 20, 1321-1345.

Ambre, J. (1985) The urinary excretion of cocaine and metabolites in humans: a kinetic analysis of published data. Journal of Analytical Toxicology, 9, 241-245.

Armbruster, D. A. \& Krolak, J. M. (1992) Screening for drugs of abuse with the Roche On TRAK assays. Journal of Analytical Toxicology, 18, 172-5.

Astley, S. J. \& Little, R. E. (1990) Maternal marijuana use during lactation and infant development at one year. Neurotoxicology and Teratology, 12, 161-163.

Atkinson, H. C. \& Begg, E. J. (1990) Prediction of drug distribution into human milk from physicochemical characteristics. Clinical Pharmacokinetics, 18, 151-167.

Batey, R. G., Patterson, T. \& Sanders, F. (1990) Practical issues in the methadone management of pregnant heroin users. Drug and Alcohol Review, 9, 303-310.

Blank, D. L. \& Kidwell, D. A. (1995) Decontamination procedures for drugs of abuse in hair: are they sufficient? Forensic Science International, 5, 13-38.

Braithwaite, R. A., Jarvie, D. R., Minty, P. S. B., et al (1995) Screening for drugs of abuse. I: Opiates, amphetamines and cocaine. Annals of Clinical Biochemistry, $32,123-53$

Brunk, S. D. (1988) False negative GC/MS assay for carboxy THC due to ibuprofen interference. Journal of Analytical Toxicology, 12, 290-295.

Burns, M. \& Baselt, R. C. (1995) Monitoring drug use with a sweat patch: an experiment with cocaine. Journal of Analytical Toxicology, 19, 41-48.

Cone, E. J. (1993) Saliva testing for drugs of abuse. Annals of the New York Academy of Science, 20, 91-127.

- (1996) Mechanisms of drug incorporation into hair. Therapeutic Drug Monitoring, 18, 438-443.

- Welch, P., Paul, B. D., et al (1991) Forensic drug testing for opiates. III. Urinary excretion rates of morphine and codeine following codeine administration. Journal of Analytical Toxicology, 15, 161-166.

-, Dickerson, S., Paul, B. D., et al (1992) Forensic drug testing for opiates. IV. Analytical sensitivity, specificity, and accuracy of commercial urine opiate immunoassays. Journal Analytical Toxicology, 16, 72-78.

Coombs, R. H. \& West, L. J. (eds) (1991) Drug Testing: Issues and Options. Oxford: Oxford University Press.

Cowan, J. M. Jr, Weathermon, A., McCutcheon, J. R., et al (1996) Determination of volume of distribution for ethanol in male and female subjects. Journal of Analytical Toxicology, 20, 287-290.

Curvall, M., Elwin, C. E., Kazemi-Vala, E., et al (1990) The pharmacokinetics of cotinine in plasma and saliva from non-smoking healthy volunteers. European Journal of Clinical Pharmacology, 38, 281-284

Dackis, C. A., Pottash, A. L. C., Annitto, W., et al (1982) Persistence of urinary marijuana levels after supervised abstinence. American Journal of Psychiatry, 139, 1196-1198.

Dickson, P. H., Lind, A., Studts, P., et al (1994) The routine analysis of breast milk for drugs abuse in a clinical toxicology laboratory. Journal of Forensic Sciences, 39, 207214.

Dupont, R. L. \& Baumgartner, W. A. (1995) Drug testing by urine and hair analysis: complementary features and scientific issues. Forensic Science International, 70, 63-76.
Eliopoulos, C., Klein, J., Chitayat, D., et al (1996) Nicotine and cotinine in maternal and neonatal hair as markers of gestational smoking. Clinical Investigations in Medicine, 19, 231-242.

Fay, J., Fogerson, R. Schoendorfer, D., et al (1996) Detection of methamphetamine in sweat by EIA and GC-MS. Journal of Analytical Toxicology, 20, 398-403.

Flanagan, R. J. (1995) The poisoned patient: the role of the laboratory. British Journal of Biomedical Science, 52, 202-213.

Fogersom, R., Schoendorfer, D., Fay, J., et al (1997) Qualitative detection of opiates in sweat by EIA and GCMS. Journal of Analytical Toxicology, 21, 451-458.

George, S. (1998) The poppy-seed defence, Syva Drug Monitor, $3,1-3$

George, S. \& Braithwaite, R. A. (1995) A preliminary evaluation of five rapid detection kits for on-site drugs of abuse screening. Addiction, 90, 227-232.

Gorodischer, R., Burtin, P., Hwang, P., et al (1994) Saliva versus blood sampling for therapeutic drug monitoring in children: patient and parental preferences and an economic analysis. Therapeutic Drug Monitoring, 16, 437-443.

Grote, C. \& Pawliszyn, J. (1997) Solid-phase microextraction for the analysis of human breath. Annals of Chemistry, 69, 589-596.

Haeckel, R. \& Hanecke, P. (1996) Application of saliva for drug monitoring. An in-vivo model for transmembrane transport. European Journal of Chemistry and Clinical Biochemistry, 34, 171-191.

Hanks, G. W. (1987) The clinical usefulness of agonistantagonist opioid analgesics in chronic pain. Drug and Alcohol Dependence, 20, 339-347.

Huestis, M. A. (1996) Judicial acceptance of hair tests for substances of abuse in the United States courts: scientific, forensic, and ethical aspects. Therapentic Drug Monitoring, 18, 456-459.

Huestis, M. A. \& Cone, E. J. (1998) Alternative testing matrices. In Drug Abuse Handbook (ed. S. Karch), pp. 799857. London: CRC Press.

Hunt, C. A. \& Jones, R. T. (1980) Tolerance and deposition of tetrahydrocannabinol in man. Journal Pharmacology $\&$ Experimental Therapeutics, 215, 35-44.

Jenkins, A. J., Darwin, W. D., Huestis, M. A., et al (1995) Validity testing of the accuPINCH THC test. Journal of Analytical Toxicology, 19, 5-12.

-, Mills, L. C., Darwin, W. D., et al (1993) Validity testing of the EZ-SCREEN cannabinoid test. Journal of Analytical Toxicology, 17, 292-298.

Joseph, R. E. Jr, Su, T. P. \& Cone, E .J. (1996) In vitro binding studies of drugs to hair: influence of melanin and lipids on cocaine binding to Caucasoid and Africoid hair. Journal of Analytical Toxicology, 20, 338-344.

Karlsson, L. \& Strom, M. (1988) Laboratory evaluation of the TDX assay for detection of cannabinoids in urine from prison inmates. Journal of Analytical Toxicology, 12, 319-321.

$\mathrm{Kim}, \mathrm{H}$. J. \& Cerceo, E. (1976) Interference by $\mathrm{NaCl}$ with the EMIT method of analysis for drugs of abuse. Clinical Chemistry, 22, 1935-1936.

Kintz, P. (1996) Drug testing in addicts: a comparison between urine, sweat, and hair. Therapeutic Drug Monitoring, 18, 450-455.

-, Tracqui, A. \& Mangin, P. (1992) Detection of drugs in human hair for clinical and forensic applications. International Journal of Legal Medicine, 105, 1-4.

- Cirimele, V. \& Mangin, P. (1995) Testing human hair for cannabis. II. Identification of THC-COOH by GC-MS-NCI as a unique proof. Journal of Forensic Science, 40, 619-622.

-, Tracqui, A., Mangim, P., Edel, Y. (1996a) Sweat testing in opioid users with a sweat patch. Journal of Analytical Toxicology, 20, 393-397.

-, - Jamey, C., et al (1996b). Detection of codeine and phenobarbital in sweat collected with a sweat patch. Journal of Analytical Toxicology, 20, 197-201.

-, - Marzullo, C., et al (1998) Enantioselective analysis of methadone in sweat as monitored by liquid chromatography spray-mass spectrometry. Therapeutic Drug Monitoring, 20, 35-40. 
Klein, J., Forman, R., Eliopoulos, C., et al (1994) A method for simultaneous measurement of cocaine and nicotine in neonatal hair. Therapeutic Drug Monitoring, 16 67-70.

Law, B., Mason, P. A., Moffat, A. C., et al (1984) Forensic aspects of the metabolism and excretion of cannabinoids following oral ingestion of cannabis resin. Journal of Pharmacy and Pharmacology, 36, 289-294.

Lemberger, L., Tamarkin, N. R. \& Alexrod, J. (1971) D'Tetrahydrocannabinol: Metabolism and disposition in long term marijuana smokers. Science, 178, 72-74.

Lewis, D., Moore, C., Morrissey, P., et al (1997) Determination of drug exposure using hair: application to child protective cases. Forensic Science International, 17, 123-128.

Linder, M. W. \& Valdes R., Jr (1994) Mechanism and elimination of aspirin induced interference in Emit II d.a.u. assays. Clinical Chemistry, 40, 1512-1515

Long, K. L. (1989) The discovery process in drug use testing litigation. Journal of Forensic Science, 34, 1454-1470.

Macdonald, D.I. (1990) The Medical Review Officer. Journal of Psychoactive Drugs, 22, 429-434.

Mack, G., Thomas, D., Giles, W., et al (1991) Methadone levels and neonatal withdrawal. Journal of Paediatrics \& Child Health, 27, 96-100.

Manolis, A., McBurney, L. J. \& Bobbie, B. A. (1983) The detection of delta-9-tetrahydro-cannabinol in the breath of human subjects. Clinical Biochemistry, 16, 229-232.

Marsh, A. (1997) Hair analysis for drugs of abuse. Syva Drug Monitor, 2, 1-4.

Martin, J. C., Barr, H. M., Martin, D. C., et al (1996) Neonatal neurobehavioral outcome following prenatal exposure to cocaine. Neu rotoxicology and Teratology, 18, 617-25.

Moeller, M. R. (1996) Hair analysis as evidence in forensic cases. Therapeutic Drug Monitoring, 18, 444-449.

Moffat, A. C., Jackson, J. V., Moss, M. S., et al (1986) Clarks Isolation and Identification of Drugs. London: Pharmaceutical Press.

Moore, C., Lewis, D., Becker, J., et al (1996) The determination of 11-nor-delta 9-tetrahydrocannabinol-9-carboxylic acid (THCCOOH) in meconium. Journal of Analytical Toxicology, 20, 50-51.

Nakahara, Y., Takahashi, K. \& Kikura, R. (1995) Hair analysis for drugs of abuse. X. Effect of physicochemical properties of drugs on the incorporation rates into hair. Biological Pharmacology Bulletin, 18, 1223-1227.

Osterloh, J. D. \& Becker, C.E. (1990) Chemical dependency and drug testing in the workplace. Journal of Psychoactive Drugs, 22, 407-417.

Sachs, H. (1995) Theoretical limits of the evaluation of drug concentrations in hair due to irregular hair growth. Forensi Science International, 70, 53-61.

Selavka, C. M. (1991) Poppy seed ingestion as a contributing factor to opiate-positive urinalysis results: the Pacific perspective. Journal of Forensic Sciences, 36, 685-696.

Simpson, D., Braithwaite, R. A., Jarvie, D. R., et al (1997) Screening for drugs of abuse. II: Cannabinoids, lysergic acid diethylamide, buprenorphine, methadone, barbiturates, benzodiazepines and other drugs. Annals of Clinical Biochemistry, 34, 460-510.

Skopp, G., Potsch, L., Eser, H. P., et al (1996) Preliminary practical findings on drug monitoring by a transcutaneous collection device. Journal of Forensic Science, 41, 933-937.

Steiner, E., Villen, T., Hallberg, M., et al (1984) Amphetamine secretion in breast milk. European Journal of Clinical Pharmacology, 27, 123-125.

Skolk, L. M., Coenradie, S. M., Smit, B. J., et al L. (1997) Analysis of methadone and its primary metabolite in meconium. Journal of Analytical Toxicology, 21, 154-159.

Strang, J., Black, J., Marsh, A., et al (1993) Hair analysis for drugs: technological breakthrough or ethical quagmire. Addiction, 88, 163-166.

Taylor, J. R,. Watson, I. D., Tames, F. J., et al (1998) Detection of drug use in a methadone maintenance clinic: sweat patches versus urine testing. Addiction, 93, 847-853.
Widdop, B. \& Caldwell, R. (1991) The operation of a hospital laboratory service for the detection of drugs of abuse. In The Analysis of Drugs of Abuse (ed. T. A. Gough), pp. 429452. England: John Wiley \& Sons.

Wilson, J. F., Smith, B. L., Toseland, P. A., et al (1994) External quality assessment of techniques for the detection of drugs of abuse in urine. Annals of Clinical Biochemistry, 31, 335342.

Wolff, K. \& Hay, A. W. M. (1994) Plasma methadone monitoring with methadone maintenance treatment. Drug \& Alcohol Dependence, 36, 69-71.

- Sanderson, M., Hay, A. W. M., et al (1991a) Methadone concentrations in plasma and their relationship to drug dosage. Clinical Chemistry, 37, 205-209.

-, Hay, A. \& Raistrick, D. (1991b) Methadone in saliva. Clinical Chemistry, 37, 1297-1298.

-, - Raistrick, D., et al (1992) Measuring compliance in methadone maintenance patients: use of a pharmacologic indicator to 'estimate' methadone plasma levels. Clinical Pharmacological Therapeutics, 50, 199-207.

-, Rostami-Hodjegan, A., Shires, S., et al (1997) The pharmacokinetics of methadone in healthy subjects and opiate users. British Journal of Clinical Pharmacology, 44, 325-334.

-, Farrell M., Marsden J., et al (1999) A review of biological indicators of illicit drug use, practical considerations and clinical usefulness. Addiction, in press.

Wu, A., Schmalz, J. \& Bennett, W. (1994) Identification of UrinAid-adulterated urine specimens by fluorometric analysis. Clinical Chemistry, 40, 845-846.

\section{Multiple choice questions}

\section{Regarding chain of custody:}

a chain of custody requires a witnessed urine sample

b the patient can bring a sample previously voided to the clinic

c temperature and specific gravity measurements should be taken to validate the specimen

d written confirmation of the validity of the sample from the individual voiding the specimen should be sought

e the patient can take the urine sample directly to the laboratory for immediate analysis.

2. Regarding false positive and false negative tests:

a a false negative is a negative finding in a sample from a patient known to have recently taken the drug of interest

b a false positive test result is the incorrect determination of the presence of a drug of interest

c there are commercially available preparations which can be used to produce false negative test results

$\mathrm{d}$ the clinician is only interested in true positive drug tests

e a drug taken legitimately may lead to a false positive test result in some circumstances. 
3. Concerning sample collection:

a blood is easy to collect in large volumes

b buccal cavity contamination is an important consideration when collecting saliva

c the sweat patch is advantageous because it is difficult to adulterate

d 24-hour urine sample collection is required for confirmation of drug test results

e maintaining alignment, securing and identifying the cut ends of hair is important if segmentation is required.

\section{About illicit drugs:}

a commonly misused opiates including methadone all have the same metabolic end product

b heroin is not excreted in urine

c over-the-counter medication containing amphetamine-type drugs can be easily distinguished from illicit substances using immunoassay tests

d cannabis is a short-acting drug

e cocaine (parent drug) is detectable in urine for 6-8 hours.
5. About biological indicators:

a urine is best for quantitative measurements of drugs currently active in the body

b hair provides a short-term alternative to urine

c saliva is a potential alternative to blood sampling

d urine provides a good qualitative indication of recent drug consumption

e meconium is widely used to screen for evidence of illicit maternal drug use.

\begin{tabular}{|c|c|c|c|c|}
\hline 1 & 2 & 3 & 4 & \\
\hline $\mathbf{T}$ & a $T$ & a $F$ & a 1 & $\mathbf{F}$ \\
\hline F & b $\mathrm{T}$ & b $\mathrm{T}$ & b & $\mathrm{T}$ \\
\hline $\mathbf{F}$ & c $\mathrm{T}$ & c $\mathrm{F}$ & c 1 & F \\
\hline $\mathrm{T}$ & d F & d F & d 1 & $\mathbf{F}$ \\
\hline $\mathrm{F}$ & e $\mathrm{T}$ & e $\mathrm{T}$ & e & $\mathrm{T}$ \\
\hline
\end{tabular}

\section{New from Gaskell}

\section{Offenders with Personality Disorder}

\section{Council Report 71}

This authoritative report, by an expert committee of the Royal College of Psychiatrists, describes contemporary classifications of personalitydisorders and the rating scales and structured interviews available for the diagnosis and assessment of personality disorders, particularly in forensic settings. It also discusses current knowledge of the prevalence of personality disorders, both in the general population and in prison and special hospital populations, and of life long courses of childhood conduct disorder and risk factors for and protective factors against delinquency.

The final and most important section of the report discusses the various therapies that have been employed in an attempt to enable personality disordered people to change their established ways of thinking, reacting and behaving, and to become less of a nuisance to others. It concludes that although a number of psychotherapeutic and behavioural regimes have shown encouraging results none has yet been adequately assessed, in this country or elsewhere, and that there is an urgent need for properly designed clinical trials. This report will be of particular interest to the wide range of people professionally concerned with personality disordered offenders, including lawyers, criminologists, social workers, probation officers, prison governors, psychologists, psychiatric nurses and psychiatrists themselves.

Book Sales, Royal College of Psychiatrists, 17 Belgrave Square, London SW1X 8PG. Telephone +44 (0)171 2352351 ext. 146, fax +44 (0)171 2451231. Credit card orders can be taken over the telephone.

See the latest information on College publications on the Internet at:

http://www.rcpsych.ac.uk 